\title{
Senescence-associated phenotypes in Akita diabetic mice are enhanced by absence of bradykinin B2 receptors
}

\author{
Masao Kakoki, Catherine M. Kizer, Xianwen Yi, Nobuyuki Takahashi, Hyung-Suk Kim, \\ C. Robert Bagnell, Cora-Jean S. Edgell, Nobuyo Maeda, J. Charles Jennette, and Oliver Smithies \\ Department of Pathology and Laboratory Medicine, University of North Carolina at Chapel Hill, Chapel Hill, North Carolina, USA.
}

\begin{abstract}
We have previously reported that genetically increased angiotensin-converting enzyme levels, or absence of the bradykinin $\mathrm{B} 2$ receptor, increase kidney damage in diabetic mice. We demonstrate here that this is part of a more general phenomenon - diabetes and, to a lesser degree, absence of the B2 receptor, independently but also largely additively when combined, enhance senescence-associated phenotypes in multiple tissues. Thus, at 12 months of age, indicators of senescence (alopecia, skin atrophy, kyphosis, osteoporosis, testicular atrophy, lipofuscin accumulation in renal proximal tubule and testicular Leydig cells, and apoptosis in the testis and intestine) are virtually absent in WT mice, detectable in B2 receptor-null mice, clearly apparent in mice diabetic because of a dominant mutation (Akita) in the Ins 2 gene, and most obvious in Akita diabetic plus B2 receptor-null mice. Renal expression of several genes that encode proteins associated with senescence and/or apoptosis (TGF- $\beta 1$, connective tissue growth factor, p53, $\alpha$-synuclein, and forkhead box O1) increases in the same progression. Concomitant increases occur in 8-hydroxy-2'-deoxyguanosine, point mutations and deletions in kidney mitochondrial DNA, and thiobarbituric acid-reactive substances in plasma, together with decreases in the reduced form of glutathione in erythrocytes. Thus, absence of the bradykinin B2 receptor increases the oxidative stress, mitochondrial DNA damage, and many senescence-associated phenotypes already present in untreated Akita diabetic mice.
\end{abstract}

\section{Introduction}

Genetically determined higher levels of angiotensin-converting enzyme (ACE), which do not significantly affect blood pressure or angiotensin II and aldosterone levels (1), have been correlated in several studies with an increased risk of a broad and overlapping constellation of diseases, including diabetic nephropathy (2), breast cancer (3), Alzheimer disease (4), Parkinson disease (5), congestive heart failure (6), myocardial infarction (7), and stroke (8). In the reverse direction, inhibitors of the enzyme (ACEIs) have beneficial effects beyond those attributable to changes in blood pressure in conditions such as diabetic nephropathy (9) and coronary artery disease (10). ACEIs have also been reported to have beneficial effects on the incidence of cancer (11) and Alzheimer disease (12). Thus, higher levels of ACE lead to an increased risk of developing many diseases that are also associated with senescence.

Some of the detrimental effects of higher ACE levels that have been observed in humans have been demonstrated in animal models. Thus, in agreement with the human data, mice with 1 , 2 , or 3 copies of the Ace gene and proportional plasma ACE levels have indistinguishable blood pressures and similar angiotensin II levels. Yet, when these mice are made diabetic with streptozotocin (STZ), the animals with 3 Ace genes develop markedly worse kidney function, as judged by higher urinary albumin excretion, than the animals with 1 or 2 Ace genes (13). This observation, com-

Nonstandard abbreviations used: ACE, angiotensin-converting enzyme; BK, bradykinin; CTGF, connective tissue growth factor; FoxO1, forkhead box O1; GSH, $\gamma$-glutamylcysteinylglycine; HG, high glucose; L-NAME, $N^{\omega}$-nitro-L-arginine methyl ester; mtDNA, mitochondrial DNA; NG, normal glucose; 8-OHdG, 8-hydroxy-2'-deoxyguanosine; STZ, streptozotocin; TBARS, thiobarbituric acid-reactive substance. Conflict of interest: The authors have declared that no conflict of interest exists. Citation for this article: J. Clin. Invest. 116:1302-1309 (2006). doi:10.1172/JCI26958. bined with prior experimental findings (14) and computer simulations (15) showing that modest changes in ACE levels affect the levels of its substrates much more than its products, led to the inference that the ACE substrate bradykinin is the likely mediator of the kidney effects.

Recently this inference received support from a demonstration that, by age 6 months, mice that are diabetic because of a dominant mutation (Akita) in the Ins 2 gene and also lack the bradykinin $\mathrm{B} 2$ receptor develop more severe kidney pathology than their diabetic littermates having the $\mathrm{B} 2$ receptor. Thus, the double mutants have a substantial degree of mesangial sclerosis in many renal glomeruli and exhibit overt albuminuria (equivalent to >550 $\mathrm{mg} / \mathrm{d}$ in humans) compared with a minor degree of mesangial sclerosis in some glomeruli and microalbuminuria (equivalent to $<150$ $\mathrm{mg} / \mathrm{d}$ ) in their simply Akita diabetic littermates (16). Surprisingly, as we now report, this combination of diabetes and absence of the bradykinin B2 receptor leads to a more general problem clearly detectable by 12 months of age: namely, the Akita diabetic mice that also lack the B2 receptor show enhancement of a variety of indicators of accelerated senescence that we find is already a feature of untreated Akita diabetic mice and (to a lesser extent) of B2 receptor-null mice.

\section{Results}

External indices of senescence. The first sign of accelerated senescence that we observed in our mice is illustrated in Figure 1A. By 12 months of age, mice heterozygous for the dominant Akita diabetogenic mutation in the insulin 2 gene (Ins $2^{\text {Akita/ }}$ ) and homozygous for a null mutation in the bradykinin B2 receptor gene (Bdkrb2- $\left.{ }^{--}\right)$ showed considerable alopecia. Hair loss was rare in the singly mutant (Akita diabetic or $\mathrm{B} 2$ receptor-null) and nonmutant WT mice at this 
A

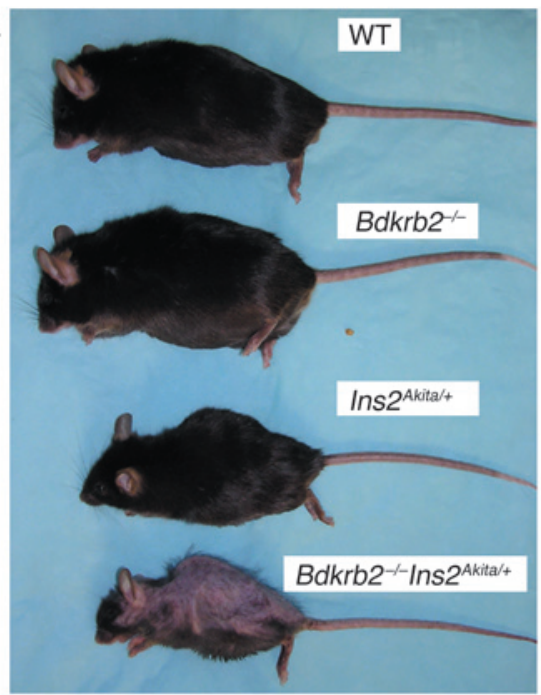

B

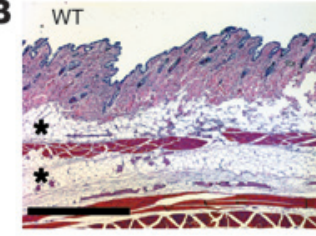
Ins2 $2^{\text {Avita/t }}$

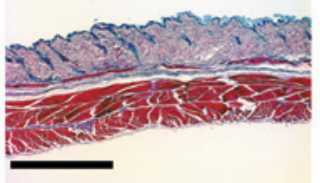

Bdkrb2 $^{--}$

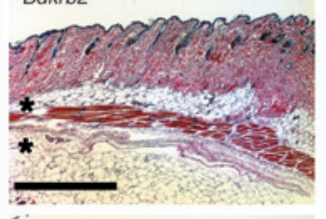

Bdkrb2-

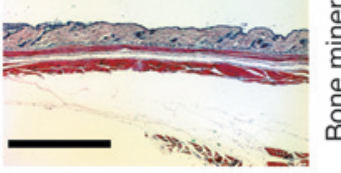

C

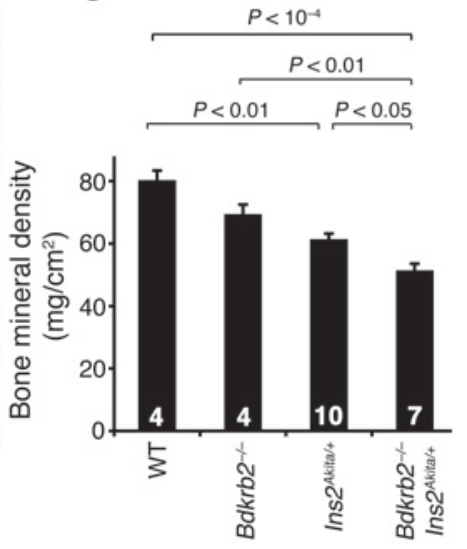

Figure 1

Appearance and associated indicators of senescence in mice at age 12 months. (A) General appearance of male mice with the following genotypes: WT at both the insulin 2 and the bradykinin B2 receptor loci, homozygous null for the B2 receptor (Bdkrb2---), heterozygous Akita for insulin 2 (Ins2 Akita/+), and doubly mutant (Bdkrb2-/-Ins2Akita/+). Kyphosis was moderate in the Akita diabetic mouse; it was severe in the doubly mutant animal, which also exhibited marked alopecia. The animals imaged were among the most severely affected of each genotype. (B) H\&E-stained abdominal skin of mice with the same 4 genotypes. Scale bars: $1 \mathrm{~mm}$. Subcutaneous fat (asterisks) was present in both nondiabetic animals but was absent in the diabetic animals. The skin of the double mutant was thin and had few hair follicles. The areas selected for imaging are representative of each genotype. (C) Bone mineral density of femurs of mice of the 4 genotypes, assessed with dual-emission $\mathrm{x}$-ray absorptiometry. Data are presented as means \pm SEM with the numbers of animals shown in white digits (see Supplemental Table 4 for 2-factor ANOVA analysis).

age (Figure 1A). The loss of hair in the double mutants was due to a reduction in the number of hair follicles and was accompanied by marked thinning of the dermis (Figure 1B). Both types of diabetic mice (singly mutant Akita and doubly mutant Akita B2 receptornull) had essentially no subcutaneous fat, although subcutaneous fat was normal in the 12-month-old nondiabetic mice (B2 receptornull and WT). The lifespans of the mice were progressively shorter (Supplemental Figure 1; supplemental material available online with this article; doi:10.1172/JCI26958DS1) in the order WT > B2 receptor-null > Akita diabetic > Akita diabetic B2 receptor-null (909 \pm 45 days, $755 \pm 23$ days, $373 \pm 30$ days, and $246 \pm 25$ days, respectively), and the effects on the lifespans of diabetic and B2 receptor-null mice were highly significant $(P<0.0001$ and $P<0.0001$, respectively, by 2 -factor ANOVA). Diabetes and absence of the B2 receptor, when combined, led to the shortest lifespan $(P<0.01$ for double mutant versus all other groups, by Tukey-Kramer honestly significant difference [HSD]) but showed no evidence of interaction ( $P$ of interaction $=0.65$ by 2 -factor ANOVA) .

The double mutants also exhibited marked kyphosis (Figure 1A), present to a lesser degree in singly mutant Akita diabetic animals but rare in B2 receptor-null mice and absent in WT mice at this age. Kyphosis is often a manifestation of osteoporosis, and Figure $1 \mathrm{C}$ shows that femur mineral bone density decreased progressively again in the order WT > B2 receptor-null > Akita diabetic > Akita diabetic $\mathrm{B} 2$ receptor-null. Diabetes and absence of the $\mathrm{B} 2$ receptor each affected bone density $(P<0.0001$ and $P<0.01$, respectively; Supplemental Table 4) and, when combined, added to the severity of this phenotype but showed no evidence of interaction ( $P$ of interaction $=0.2$ ). The doubly mutant mice had the most severe kyphosis and the greatest reduction in bone density $(P<0.05$ versus other groups, by Tukey-Kramer HSD).
Cellular indices of senescence/apoptosis. These external signs of accelerated senescence prompted us to look at other indicators of aging in the 4 types of mice, including decreases in spermatogenesis. Figure $2 \mathrm{~A}$ shows that all spermatic cords appeared normal in the WT and $\mathrm{B} 2$ receptor-null animals at 12 months of age. A loss of spermatogenesis was visible in some cords (outside the dotted red line) in the Akita diabetic animals. Atrophic changes were severe in the double mutants (Akita diabetic B2 receptor-null).

We also observed numerous vacuoles containing intracellular accumulations of brown pigmented material in the testicular Leydig cells of the Akita diabetic B2 receptor-null mice (Figure 2B). They were present to a somewhat lesser degree in the simply diabetic animals, were much fewer in the $\mathrm{B} 2$ receptor-null mice, and were absent in the WT mice. This same type of material accumulated in the renal proximal tubule cells (Figure 3A) of the Akita diabetic B2 receptor-null mice, and to a lesser degree in the simply diabetic animals; it was virtually absent in nondiabetic animals lacking the $\mathrm{B} 2$ receptor and in the WT mice. Electron microscopy of the renal proximal tubule cells showed that they contained phagolysosomes with laminated lipid debris consistent with their being autophagolysosomes (Figure 3B). Intracellular accumulations in autophagolysosomes of lipid and protein debris, including lipofuscin, are known indicators of cellular senescence (17), oxidative stress, and increased mitochondrial turnover (18). Finding these deposits suggests that mitochondrial damage plays an important role in the graded deterioration of renal function described previously in 6-month-old Akita diabetic and doubly mutant mice (16), and in the graded loss of testicular function described here in the 12-month-old mice (Figure 2A).

A senescence-associated phenotype associated with increased apoptosis, particularly in cells with a rapid cellular turnover, 
A

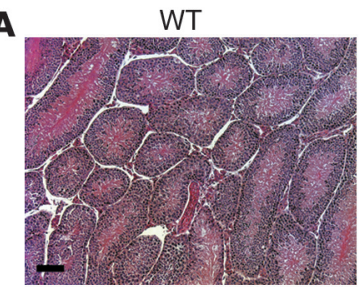
Ins2 $2^{\text {Akita/+ }}$

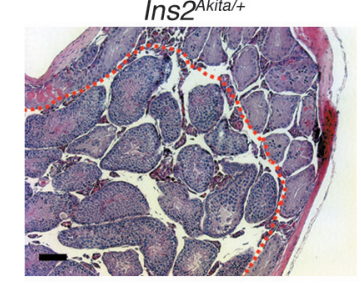

B

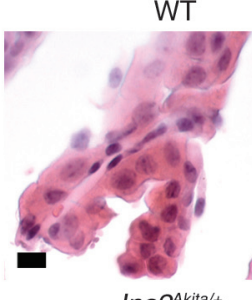

Ins2 $2^{\text {Akita/ }}$

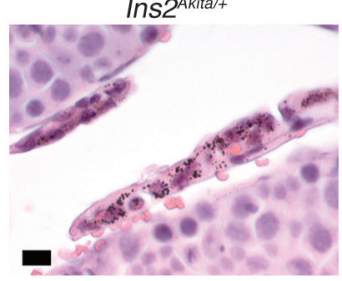

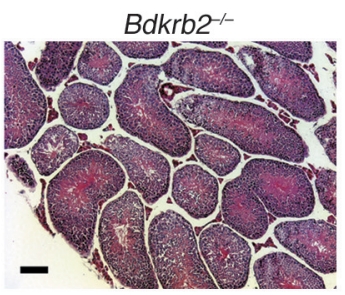

Bdkrb2-1-Ins2 $2^{\text {Akita/+ }}$

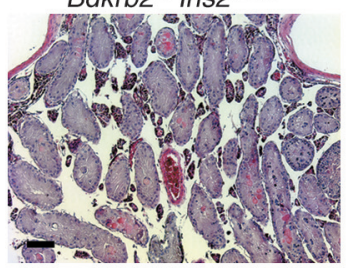

$B d k r b 2^{-1-}$

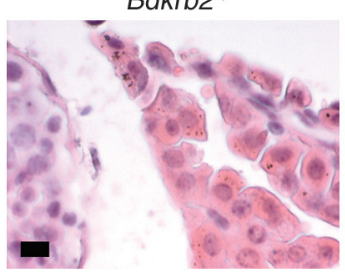

Bdkrb2--Ins2 Akita/t

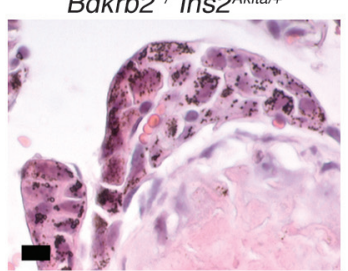

has recently been described in mice carrying a mutator allele of mitochondrial DNA polymerase $\gamma(19)$, and in mice with short telomeres (20). We therefore asked whether the pathological differences observed in the testes (Figure 2) and kidneys (Figure 3) of our 12-month-old mice were paralleled by differences in the extent of apoptosis in these same tissues, as assayed by TUNEL staining. We found that the degree of apoptosis approximately paralleled the observed gradation in loss of spermatogenesis in

\section{Figure 2}

Testes of 12-month-old mice of the 4 genotypes. (A) Low-magnification H\&E-stained testes. Loss of spermatogonia was severe in the double mutant. Some spermatic cords in the Akita diabetic mouse were aspermic (outside the dotted red line). The testes of the B2 receptor-null and WT mice had normal spermatic cords. (B) High-magnification H\&E-stained testes of 12-month-old mice. There were numerous intracellular pigmented vacuoles in the Leydig cells of the doubly mutant mouse. Pigmented vacuoles in Leydig cells were also present to a somewhat lesser degree in the Akita diabetic mouse. They were sparse in the B2-null mouse, and absent in the WT mouse. The areas selected for imaging in $\mathbf{A}$ and $\mathbf{B}$ are representative of each genotype. Scale bars: $100 \mu \mathrm{m}(\mathbf{A}), 10 \mu \mathrm{m}(\mathbf{B})$.

the 4 types of mice. Diabetes and absence of the $\mathrm{B} 2$ receptor both had highly significant and strongly interacting effects on the frequency of the apoptotic cells in the seminiferous tubules, the cells of which had rapid turnover (Supplemental Figure 2, A and B, and Supplemental Table 4). We also observed a very similar gradation in the frequency of apoptotic cells in intestinal villi, another site of rapid cellular turnover (Supplemental Figure 3, A and B, and Supplemental Table 4).

Senescence-associated gene expression in vivo. Aging is accompanied by changes in gene expression. For example, the expression of TGF- $\beta 1$ and of connective tissue growth factor (CTGF) is enhanced in old animals and in late passages of cultured cells (21). Increases in the expression of these apoptosis-related genes $(22,23)$ has also been implicated in changes that accompany fibrogenesis in diabetes (24). Similarly, increased expression of the apoptosis-related genes p53 (23), forkhead box O1 (FoxO1) (25), and $\alpha$-synuclein (26) has been reported in senescent cells and/or animals $(21,27,28)$. Table 1 shows that the expression of all these genes was increased relative to WT in the kidneys of the B2 receptor-null animals (6\% to $47 \%$ greater than WT), even more in the Akita diabetic animals (57\% to $114 \%$ ), and still more in the double mutants (128\% to $325 \%)$. The effects of diabetes on expression $(P<0.0001$ for all the genes; Supplemental Table 4 ) and of absence of the $\mathrm{B} 2$ receptor on expression $(P<0.05$ for all the genes except CTGF, $P=0.06$; Supplemental Table 4) were both significant with no evidence of interaction, and the double mutant was significantly more affected than any of the others $(P<0.05$ versus other groups).

\section{Figure 3}

Kidneys of 12-month-old mice of the 4 genotypes. (A) PAS-stained kidneys. There were numerous intracellular pigmented vacuoles in the cytoplasm of the proximal tubular epithelial cells of the doubly mutant mouse. Pigmented vacuoles were present to a lesser degree in the proximal tubules of the Akita diabetic mouse with intact B2 receptors but were absent in the $\mathrm{B} 2$ receptor-null and WT nondiabetic mice. The areas selected for imaging are representative of each genotype. (B) Transmission electron micrograph of a renal proximal tubule cell from a doubly mutant mouse. Note the numerous lipid debris-containing phagolysosomes with focal laminations (arrows). Scale bars: $10 \mu \mathrm{m}(\mathbf{A}), 1 \mu \mathrm{m}$ (B).
A

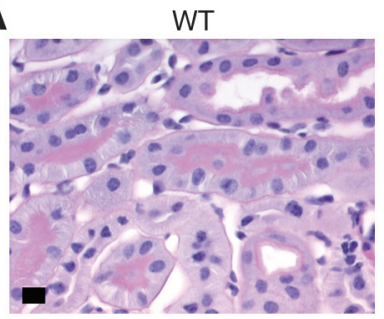
Ins2 $2^{\text {Akita/t }}$

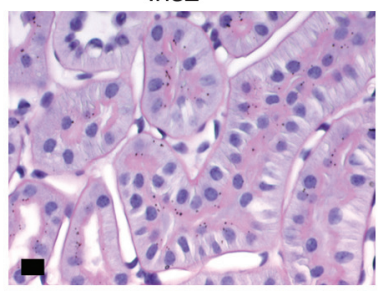

Bdkrb2--

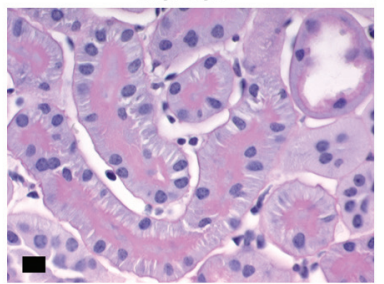

Bdkrb2--Ins2 $2^{\text {Akita/+ }}$

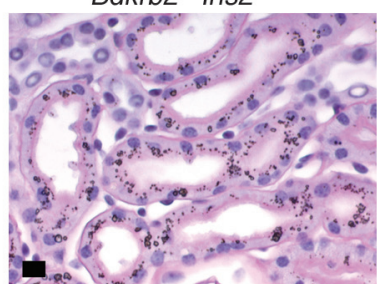

B

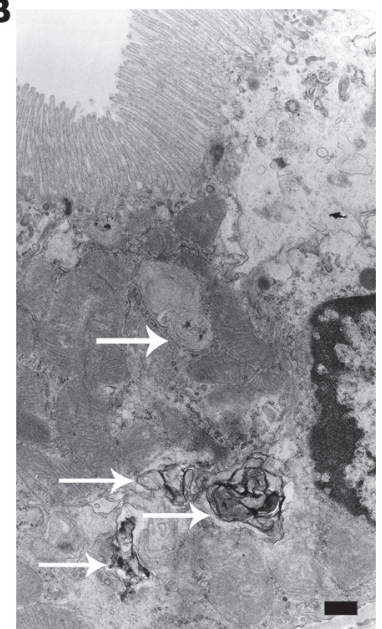


Table 1

mRNAs in the kidneys of 4 types of animals at age 12 months

\begin{tabular}{lcclc}
\hline & & & & \\
& WT & Bdkrb2-- & Ins2 Akita/+ $^{-}$ & Bdkrb2-/Ins2 $^{\text {Akita/+ }}$ \\
TGF- $\beta 1$ & $16.6 \pm 4.4$ & $18.7 \pm 4.4$ & $39.9 \pm 3.3^{\mathrm{A}}$ & $58.1 \pm 3.6^{\mathrm{A}, \mathrm{B}}$ \\
& $(100 \pm 27)$ & $(113 \pm 27)$ & $(240 \pm 20)$ & $(350 \pm 22)$ \\
CTGF & $45.9 \pm 8.4$ & $48.4 \pm 8.4$ & $78.8 \pm 6.3^{\mathrm{A}}$ & $106.7 \pm 6.8^{\mathrm{A}, \mathrm{B}}$ \\
& $(100 \pm 18)$ & $(105 \pm 18)$ & $(172 \pm 14)$ & $(233 \pm 15)$ \\
p53 & $(100 \pm 19)$ & $(141 \pm 19)^{\mathrm{A}}$ & $(175 \pm 14)^{\mathrm{A}}$ & $(242 \pm 17)^{\mathrm{A}, \mathrm{B}}$ \\
$\alpha$-Synuclein & $(100 \pm 46)$ & $(147 \pm 46)^{\mathrm{A}}$ & $(266 \pm 35)^{\mathrm{A}}$ & $(425 \pm 41)^{\mathrm{A}, \mathrm{B}}$ \\
Fox01 & $(100 \pm 21)$ & $(131 \pm 21)^{\mathrm{A}}$ & $(178 \pm 16)^{\mathrm{A}}$ & $(257 \pm 19)^{\mathrm{A}, \mathrm{B}}$ \\
\hline
\end{tabular}

Means \pm SEM are shown for assays with 4-7 animals of each genotype. Data for TGF- $\beta 1$ and CTGF are presented as picograms specific mRNA per microgram total RNA, with percentage of WT in parentheses. Data for the other genes are relative to expression of $\beta$-actin and are presented in parentheses as percentage of WT. ${ }^{A} P<0.05$ versus $W T,{ }^{B} P<0.05$ versus Ins $2^{A k i t a /+}$, by TukeyKramer HSD (see Supplemental Table 4 for 2-factor ANOVA analyses).

Oxidative stress in vivo. These several observations show that absence of the bradykinin B2 receptor increases the severity of a variety of senescence-associated changes that occur in untreated diabetic mice. The changes in kidney proximal tubule cells and the Leydig cells of the testis illustrated above are particularly informative. These 2 cell types both have high metabolic activity as judged by their mitochondrial content. They are consequently likely to be particularly vulnerable to disturbances in mitochondrial function and to oxidative stress. We therefore determined in our animals the level of thiobarbituric acid-reactive substances (TBARSs) in plasma and of reduced glutathione ( $\gamma$-glutamylcysteinylglycine; GSH) in erythrocytes, 2 reciprocal indicators of oxidative stress. The resulting data (Figure 4, A and B) show that the TBARS levels increased and the GSH levels decreased in the same progression as in the senescence-associated phenotypes. The effects of diabetes and of absence of the B2 receptor on TBARSs and GSH were both highly significant $(P<0.001$; Supplemental Table 4$)$, with no evidence of interaction $(P>0.05)$.

Mitochondrial DNA damage in vivo. Damage to mitochondrial DNA (mtDNA), including an increased content of 8-hydroxy-2'deoxyguanosine $(8-\mathrm{OHdG})$ and increased frequencies of point mutations and deletions, is a known consequence of oxidative stress, and it increases with age (29-31). Accordingly, we determined the extent of damage to mtDNA in our mice. Figure 5 (A, B, and $C$ ) shows that the incidence of these several indicators of DNA damage and senescence increased progressively in kidney mtDNA, from WT mice to B2 receptor-null mice to Akita diabetic mice to Akita diabetic B2 receptor-null mice. The effects of diabetes on all these indicators of DNA damage $(P<0.0001$; Supplemental Table $4)$ and of absence of the B2 receptor $(P<0.05)$ were both significant by 2 -factor ANOVA with no evidence of interaction, except for 8 -OHdG $(P<0.001)$. Approximately $65 \%$ of the point mutations in all groups were A:T to G:C transitions (data not shown). A similar distribution of point mutations in association with aging has been reported previously $(19,32)$.

Oxidative stress in vitro. The bradykinin $\mathrm{B} 2$ receptor acts in part via its downstream influence on NO formation. We therefore carried out in vitro experiments to determine the effects of bradykinin (BK) on oxidative stress induced by high glucose, and to determine the effects on the induced oxidative stress of inhibiting NO production. In these experiments we used EA.hy926 vascular endothelial cells (33) and a fluorochrome that localizes in mitochondria and fluoresces when oxidized. Figure 6 (A and B) shows that the fluorescence of the endothelial cells cultured in a high-glucose (HG) medium, containing glucose at the same concentration at which it occurs in the plasma of our diabetic mice, $750 \mathrm{mg} / \mathrm{dl}$, increased significantly relative to the fluorescence of cells cultured at a normal glucose (NG) concentration, 100 $\mathrm{mg} / \mathrm{dl}(P<0.01$, by Tukey-Kramer HSD after 1 -way ANOVA), indicating that $\mathrm{HG}$ increases mitochondrial oxidative stress. Addition of BK to the HG medium drastically decreased the fluorescence $(P<0.0001)$, indicating that BK markedly decreases this oxidative stress. The fluorescence returned almost to that seen with HG alone when $N^{\omega}$-nitro-L-arginine methyl ester (L-NAME), an inhibitor of the NO synthases, was added to the HG plus BK medium $(P>0.05)$, indicating that the ameliorating effect of BK is mediated largely by NO.

\section{Discussion}

Diabetes mellitus in humans is the leading cause of endstage renal disease (34), and increased urinary albumin excretion is a well-established predictor of its development. Urinary albumin also increases in nondiabetic subjects with aging (35). Diabetes mellitus is a risk factor for many other conditions that also increase in frequency in nondiabetic individuals as they age. It is a risk factor for myocardial infarction (relative risk, 6.9) (36), dilated cardiomyopathy (relative risk, 3.0) (37), sudden cardiac death (relative risk, 4.0) (38), stroke (relative risk, 5.8) (39), Alzheimer disease (relative risk, 1.9) (40), and bone fractures (relative risk, 1.3) (41). It is a risk factor for cancer of the liver (relative risk, 2.2) (37), stomach (relative risk, 2.3) (42), colorectum (relative risk, 3.2) (37), pancreas (relative risk, 1.5) (37), bladder (relative risk, 1.4) (37), cervix (relative risk, 1.6) (42), breast (relative risk, 1.3) (37), and endometrium (relative risk, 2.7) (42). Aging and diabetes are also independent risk factors for retinal macular degeneration (43) and distal polyneuropathy (44). Thus, diabetes mellitus affects the risk of developing many conditions that are associated with senescence.

The experimental data that we have presented demonstrate that, in 12-month-old mice, absence of the bradykinin B2 receptor combined with diabetes due to the Akita mutation markedly
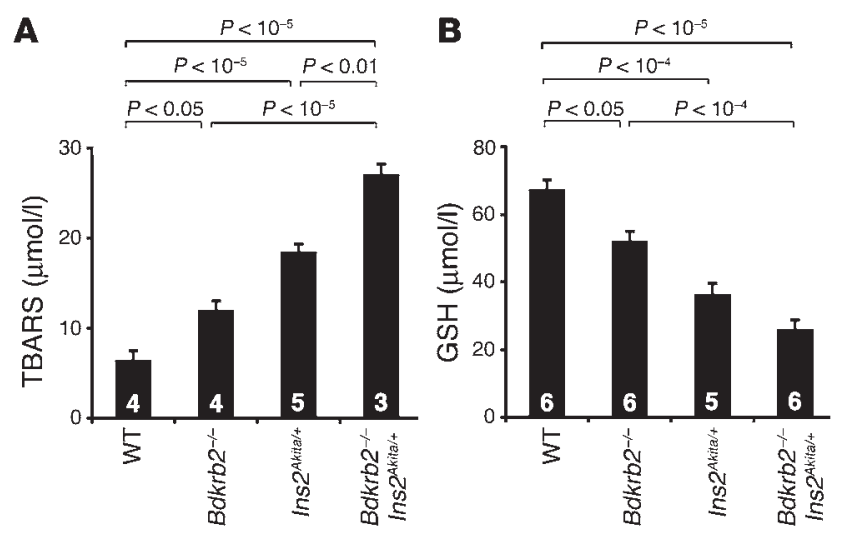

Figure 4

Indices of oxidative stress in 12-month-old mice of the 4 genotypes. (A) Plasma levels of TBARSs. (B) Concentration of reduced glutathione (GSH) in erythrocytes. Data are presented as means \pm SEM with the numbers of animals shown in white digits (see Supplemental Table 4 for 2-factor ANOVA analysis). 

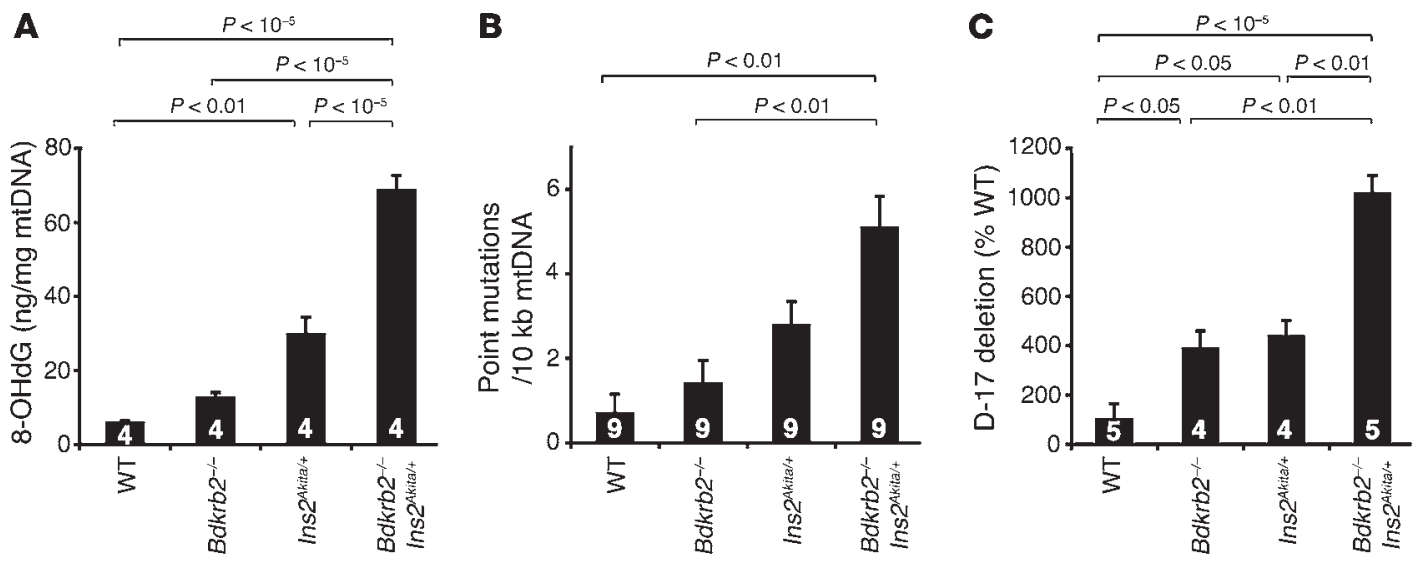

Figure 5

Alterations in mtDNA in 12-month-old mice of the 4 genotypes. (A) 8-OHdG content in renal cortical mtDNA. (B) Frequencies of point mutations in the cytochrome $b$ gene in the mtDNA used in A. (C) Relative proportion of D-17 deletions (as percentage of WT) in the same DNA, determined by quantitative PCR. Data are presented as means \pm SEM with the numbers of animals shown in white digits (see Supplemental Table 4 for 2-factor ANOVA analysis).

increases the severity of a wide range of senescence-associated phenotypic abnormalities that occur to a lesser degree in mice that are simply Akita diabetic. Although the extent of senescence-associated phenotypes in the simply Akita diabetic mice is much less than in the double mutants, it is nevertheless more severe than that normally seen in most human insulin-dependent diabetics in advanced societies. This is not unexpected, because the severe diabetes of the Akita mice is not treated, and because the mice are inbred. In contrast, insulin-dependent human diabetics are treated with insulin in developed countries and have much better genetic backgrounds (they are not inbred). Some comment is also required on our choice of the dominant Akita mutation to induce diabetes, rather than induction by STZ treatment. One reason is that the Akita-induced diabetes has a better-defined etiology (endoplasmic reticulum stress and proteotoxicity in the pancreatic $\beta$ cells) than the diabetes due to STZ, and another reason is that the diabetes does not regress. Additionally, the genetic model gives severalfold tighter data than the chemical model, and the nephropathy that develops in the Akita diabetic mice is more like that seen in human diabetics than is the nephropathy that develops in mice with chemically induced diabetes (45). Consequently, our present findings regarding the factors responsible for the development of senescence-related phenotypes in diabetic mice, and their enhancement by absence of the bradykinin $\mathrm{B} 2$ receptor, bear strongly on the complications of diabetes in humans and their similarities to the effects of age in nondiabetic individuals.

For more than 50 years it has been proposed that free radicals generated during normal or abnormal oxidative metabolism can cause sufficient cumulative damage to cells to lead to aging (46); and it has recently been shown that mitochondrial overproduction of superoxide activates major pathways of hyperglycemia-induced cellular damage (47). In our mice we find that the incidence of overt signs of senescence (alopecia, skin atrophy, kyphosis, osteoporosis, autophagosomic inclusions in renal tubule cells and testicular Leydig cells, testicular atrophy) and the incidence of changes in the expression of genes implicated in aging (TGF- $\beta 1$, CTGF, p53, FoxO1, and $\alpha$-synuclein) increase in parallel with increases in ROS (plasma TBARSs) and reciprocal decreases in reductive species (erythrocyte GSH). The content of 8-OHdG in mtDNA increases in the same progression as the metabolic indicators of oxidative stress and the senescence-related phenotypes, as does the frequency of point mutations. However, we find that the majority

\section{Figure 6}

Oxidative stress in vitro. (A) Mitochondrial oxidation (assessed by MitoTracker Red $\mathrm{CM}-\mathrm{H}_{2} \mathrm{XRos}$ fluorescence) in EA.hy926 vascular endothelial cells cultured in the presence of normal glucose (NG), high glucose $(\mathrm{HG})$, $\mathrm{HG}$ plus $1 \mu \mathrm{M}$ bradykinin (BK), or HG plus BK plus $1 \mathrm{mM}$ L-NAME. $B K$ reduced the oxidative signal seen in $\mathrm{HG}$; this reduction was largely abolished by the NOS inhibitor L-NAME. Scale bars: $100 \mu \mathrm{m}$. (B) Quantitative analysis of MitoTracker Red fluorescence from single cells under the 4 culture conditions used in $\mathbf{A}$. The data are means \pm SEM with the numbers of cells shown in white digits.
A
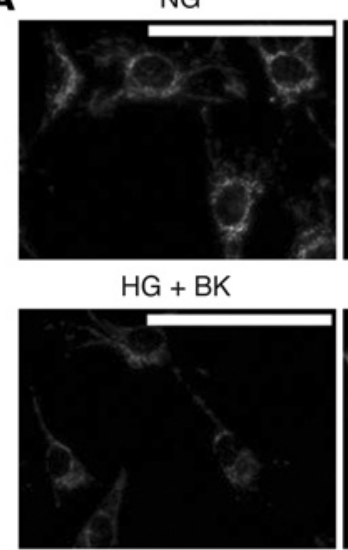

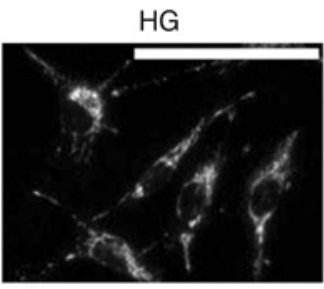

$\mathrm{HG}+\mathrm{BK}+\mathrm{L}-\mathrm{NAME}$

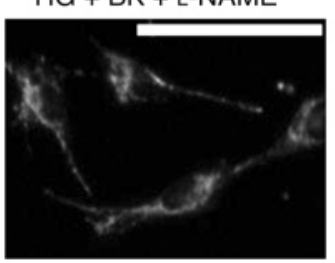

B

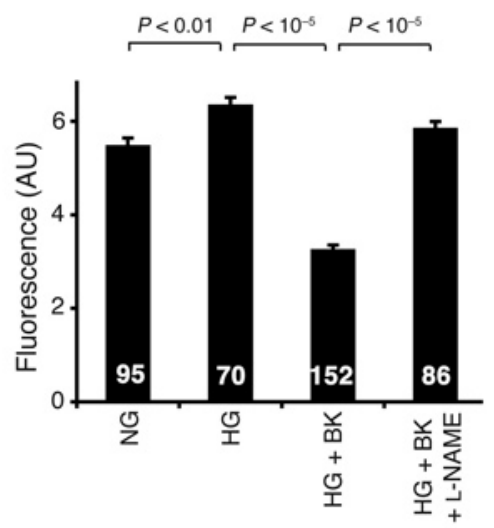


of the additional point mutations are transitions, as also reported by others in aging mice $(19,32)$, whereas 8 -OHdG leads mainly to transversions (48). We conclude that, although the increased level of 8-OHdG is a well-known indicator of oxidative stress, the point mutations we observe are unlikely to be a direct consequence of this modified base. However, we cannot exclude the possibility that the approximately 10 -fold increase in the level of 8-OHdG over the WT level in the mtDNA of our double mutants affects their senescence-associated phenotype, even though premature aging was not reported in mice with an approximately 3 -fold increase in the level of mitochondrial 8-OHdG over the WT level, caused by heterozygosity for disruption of the Sod2 gene (49).

Two recent reports $(19,50)$ are relevant to our present work. Both report that mice with a defective proofreading function in their mitochondrial DNA polymerase $\gamma$ develop senescence-associated phenotypes similar to those that we observe (including alopecia, kyphosis, osteoporosis, and reduced fertility). The mutants have an increased frequency of mutations in their mtDNA and an increased induction of apoptotic markers, particularly in tissues characterized by rapid cellular turnover, but do not have increased oxidative stress (19). However, this result does not exclude the possibility that chronic and severe oxidative stress can also lead to mitochondrial damage - including mutations like those reported in the polymerase $\gamma$ mutants - that is sufficient to induce apoptosis in susceptible tissues. Thus, diabetes and absence of the B2 receptor independently and largely additively increase oxidative stress in our mice. Diabetes and absence of the B2 receptor independently - and even more in combination - increase the presence of mitochondrial debris in testicular Leydig cells and renal tubule cells. And diabetes and absence of the $\mathrm{B} 2$ receptor each increase the number of apoptotic cells in testicular seminiferous tubules and in intestinal villi of our mice, with the combined effects of the 2 factors being significantly greater than the sum of their individual effects $(P$ of interaction $<0.01)$. We infer from these observations that the graded oxidative stress in our mutant mice increases mitochondrial damage, readily detectable in metabolically active cells, and that this damage can reach a level sufficient to induce apoptosis in some actively dividing cells.

The experiments with endothelial cells that we have described here establish a connection among oxidative stress, diabetes, BK, and NO. High glucose induces an increase in oxidative stress in these cells that is inhibited by BK largely by an NO pathway. Experiments by others (51) have shown that oxidative stress induced by high glucose in cultured endothelial cells is ameliorated by an ACE inhibitor, and that this amelioration is blocked by a $\mathrm{B} 2$ receptor antagonist, but not by a B1 receptor antagonist. Thus the in vitro ameliorating effects on oxidative stress of BK and of ACE inhibitors are mediated at least in part through a common pathway that includes the bradykinin B2 receptor and probably NO.

A further clue to the possible chain of events leading to our results is provided by studies showing that endogenously produced $\mathrm{NO}$ regulated by $\mathrm{Ca}^{++}$influx and acting through cytochrome oxidase, the enzyme responsible for approximately $90 \%$ of oxygen consumption, is probably responsible for the physiological regulation of respiration in endothelial and other cells $(51,52)$; when oxygen tension is low, NO inhibits aerobic metabolism. NO regulates cardiac oxygen consumption through a bradykinin-receptor mechanism, and this physiological mechanism is absent in B2 receptor-null mice (53). Endothelial NOS plays an essential role in the regulation of renal oxygen consumption by $\mathrm{NO}$ (54), and
NO (after inhibiting cytochrome oxidase) switches on glycolysis by activating the master regulator of glycolysis, PFK1 (55). Accordingly, we suggest that a decrease in intracellular NO, caused either by reduced BK levels or by absence of the bradykinin $\mathrm{B} 2$ receptor (56), may impair this check on aerobic metabolism, with a consequent increase in ROS and oxidative stress. Even in the absence of diabetes, BK is important in the context of accelerated senescence, as indicated in Supplemental Figure 4, which shows that if mice lack the B2 receptor they develop alopecia and kyphosis by 2 years of age comparable to that seen in the B2-null Akita diabetic mice at 1 year of age, and not seen in WT mice of the same age. This is consistent with a report showing aging phenotypes in the hearts of mice lacking the B2 receptor (57).

In summary, we have demonstrated that absence of the bradykinin B2 receptor increases the incidence of many detrimental factors also apparent in untreated Akita diabetic mice, including oxidative stress, 8-OHdG content, mutations in mtDNA, mitochondrial turnover, the expression of several senescence/apoptosis-related genes, and the occurrence of apoptosis in testicular seminiferous tubules and intestinal villi. Together these increases caused by absence of the bradykinin B2 receptor culminate in a marked enhancement of a variety of senescence-associated phenotypes that are already present to a lesser degree in the untreated diabetic mice.

\section{Methods}

Animals. Mice were purchased from Jackson Laboratory and bred in our animal facility. Mice having the null allele for the bradykinin B2 receptor (Bdkrb2-/-) (58) were backcrossed to WT C57BL/6J at least 6 times before mating to mice heterozygous for the Akita mutation (Ins $2^{\text {Akita/ }+}$ ) (59), which already have the C57BL/6J genetic background. All experiments were approved by the University of North Carolina Institutional Animal Care and Use Committee. Bone mineral density in femurs was measured with dual-emission $x$-ray absorptiometry (LUNAR PIXImus 2; GE Healthcare) in the Body Composition Core of the Clinical Nutrition Research Center at the University of North Carolina. The histopathological data were obtained with tissues from at least 4 mice of each genotype. The specific images shown were selected as representative of the tissue from each genotype. The images illustrating the general appearance of the animals were of the severely affected mice of each of the genotypes. For assays where samples could be stored before processing, age-matched animals were euthanized at different times, but the assays themselves were performed together in 1 batch. For quantitative measurements inherently sensitive to inadvertent experimental variations (for example, the assays for TBARSs), all samples were collected on the same day and measurements were made together.

$8-O H d G$ levels and the frequency of point mutations in mtDNA. Mitochondrial DNA (mtDNA) was extracted from whole kidney using an mtDNA Extractor CT kit (Wako Pure Chemicals Industries Ltd.). The 8-OHdG levels in DNase I-digested mtDNA were determined by ELISA with a kit from the Japan Institute for the Control of Aging. To determine the sequence of the mitochondrial cytochrome $b$ gene (GenBank accession number gi33115104) in our mtDNA samples, we amplified its coding region with Tgo DNA polymerase (Roche Applied Science) using primers shown in Supplemental Table 1. The PCR-amplified fragments were subcloned into pBluescript II (Stratagene) and sequenced with M13 primers.

Quantification of mtDNA deletion mutants. Large deletions between 2 homologous sequences in mtDNA have been reported in aged mice (31). We therefore assayed the most common deletion mutation, D-17, in our mtDNA samples using quantitative PCR with primers flanking the D-17 deletion and with template mtDNAs cut at an MluI site present in the region deleted in D-17. The sequences of primers and probes for quantifi- 
cation of the normal and D-17-deleted cytochrome $b$ gene are provided in Supplemental Table 2.

Quantitative RT-PCR. Total RNA was extracted from whole kidney, and the mRNAs for TGF- $\beta 1$, CTGF, p53, FoxO1, and $\alpha$-synuclein were assayed by quantitative RT-PCR. The sequences of the primers and probes for measurement of the expression of these genes are provided in Supplemental Table 3.

Measurement of TBARSs and GSH. The plasma levels of TBARSs were determined as described elsewhere (60). The concentration of reduced glutathione (GSH) in erythrocytes was measured with a Glutathione Assay kit (Calbiochem).

Microscopy. Fluorescence of MitoTracker Red CM- $\mathrm{H}_{2}$ XRos (Invitrogen Corp.), an index of mitochondrial oxidation, was evaluated in EA.hy926 vascular endothelial cells (33) with an IX70 inverted microscope (Olympus) equipped with a filter set for Texas red (exciter 560/55, dichroic 595, emitter 645/75; Chroma Technology Corp.). In the NG medium, glucose was at 100 $\mathrm{mg} / \mathrm{dl} \mathrm{HBSS}$ with $\mathrm{Ca}^{++}$and $\mathrm{Mg}^{++}$but without phenol red. Glucose was at 750 $\mathrm{mg} / \mathrm{dl}$ in the HG medium. BK, when used, was at $1 \mu \mathrm{M}$, and the NOS inhibitor L-NAME was at $1 \mathrm{mM}$. The endothelial cells were incubated for 60 minutes in NG, HG, HG plus BK, or HG plus BK plus L-NAME medium before addition of MitoTracker Red CM- $\mathrm{H}_{2} \mathrm{XRos}(1 \mu \mathrm{M})$. Fluorescence was recorded 15 minutes later with a SPOT RT Slider digital camera, and analyzed with SPOT version 4.0.9 (Diagnostic Instruments) and ImageJ $1.33 \mathrm{u}(\mathrm{NIH})$ software.

TUNEL assay. We performed TUNEL assay in the paraffin-embedded duodenum and testis using ApopTag Fluorescein In Situ Apoptosis Detec- tion Kit (Chemicon International). A filter set for yellow fluorescent protein (exciter 500/20, dichroic 515, emitter 535/30; Chroma Technology Corp.) was used to detect the FITC fluorescence.

Statistics. Data are expressed as means \pm SEM. To compare the effects of diabetes, of absence of the $\mathrm{B} 2$ receptor, and of their combination, we used 2-factor ANOVA with the program JMP 5.1.2 (SAS Institute Inc.; $P$ values for 2-factor ANOVA are shown in Supplemental Table 4). Post hoc pairwise comparisons were by Tukey-Kramer HSD. $P$ values less than 0.05 were considered significant.

\section{Acknowledgments}

The authors are grateful to Joseph A. Brackhan, Thomas M. Coffman, Joyce B. Harp, Kunjie Hua, Shinja Kim, Ikuko Mohri, Kinuko Suzuki, and Melissa Taylor for help. This work was supported by NIH grants HL49277, HL70523, and HL71266.

Received for publication September 23, 2005, and accepted in revised form February 14, 2006.

Address correspondence to: Oliver Smithies, Department of Pathology and Laboratory Medicine, CB \#7525, 701 BrinkhousBullitt Building, University of North Carolina at Chapel Hill, Chapel Hill, North Carolina 27599-7525, USA. Phone: (919) 966-6913; Fax: (919) 966-8800; E-mail: jenny_langenbach@ med.unc.edu.
1. Lachurie, M.L., Azizi, M., Guyene, T.T., AlhencGelas, F., and Menard, J. 1995. Angiotensin-converting enzyme gene polymorphism has no influence on the circulating renin-angiotensin-aldosterone system or blood pressure in normotensive subjects. Circulation 91:2933-2942.

2. Marre, M., et al. 1994. Relationships between angiotensin I converting enzyme gene polymorphism, plasma levels, and diabetic retinal and renal complications. Diabetes. 43:384-388.

3. Koh, W.P., et al. 2003. Angiotensin I-converting enzyme (ACE) gene polymorphism and breast cancer risk among Chinese women in Singapore. Cancer Res. 63:573-578.

4. Kehoe, P.G., et al. 1999. Variation in DCP1, encoding ACE, is associated with susceptibility to Alzheimer disease. Nat. Genet. 21:71-72.

5. Lin, J.J., Yueh, K.C., Chang, D.C., and Lin, S.Z. 2002. Association between genetic polymorphism of angiotensin-converting enzyme gene and Parkinson's disease. J. Neurol. Sci. 199:25-29.

6. Raynolds, M.V., et al. 1993. Angiotensin-converting enzyme DD genotype in patients with ischaemic or idiopathic dilated cardiomyopathy. Lancet. 342:1073-1075.

7. Cambien, F., et al. 1992. Deletion polymorphism in the gene for angiotensin-converting enzyme is a potent risk factor for myocardial infarction. Nature. 359:641-644.

8. Doi, Y., et al. 1997. Polymorphism of the angiotensin-converting enzyme (ACE) gene in patients with thrombotic brain infarction. Atherosclerosis. 132:145-150.

9. Lewis, E.J., Hunsicker, L.G., Bain, R.P., and Rohde, R.D. 1993. The effect of angiotensin-converting-enzyme inhibition on diabetic nephropathy. The Collaborative Study Group. N. Engl. J. Med. 329:1456-1462.

10. Mancini, G.B.J., et al. 1996. Angiotensin-converting enzyme inhibition with quinapril improves endothelial vasomotor dysfunction in patients with coronary artery disease. Circulation. 94:258-265.

11. Lever, A.F., et al. 1998. Do inhibitors of angiotensin-I-converting enzyme protect against risk of can- cer? Lancet. 352:179-184.

12. Ohrui, T., et al. 2004. Effects of brain-penetrating ACE inhibitors on Alzheimer disease progression. Neurology. 63:1324-1325.

13. Huang, W., et al. 2001. Genetically increased angiotensin I-converting enzyme level and renal complications in the diabetic mouse. Proc. Natl. Acad. Sci. U. S. A. 98:13330-13334.

14. Campbell, D.J., Kladis, A., and Duncan, A.M. 1994. Effects of converting enzyme inhibitors on angiotensin and bradykinin peptides. Hypertension. 23:439-449.

15. Smithies, O., Kim, H.S., Takahashi, N., and Edgell, M.H. 2000. Importance of quantitative genetic variations in the etiology of hypertension. Kidney Int. 58:2265-2280.

16. Kakoki, M., Takahashi, N., Jennette,J.C., and Smithies, O. 2004. Diabetic nephropathy is markedly enhanced in mice lacking the bradykinin B2 receptor. Proc. Natl. Acad. Sci. U. S. A. 101:13302-13305.

17. Brunk, U.T., and Terman, A. 2002. Lipofuscin: mechanisms of age-related accumulation and influence on cell function. Free Radic. Biol. Med. 33:611-619.

18. Terman, A., Dalen, H., Eaton, J.W., Neuzil, J., and Brunk, U.T. 2004. Aging of cardiac myocytes in culture. Oxidative stress, lipofuscin accumulation, and mitochondrial turnover. Ann. N. Y. Acad. Sci. 1019:70-77.

19. Kujoth, G.C., et al. 2005. Mitochondrial DNA mutations, oxidative stress, and apoptosis in mammalian aging. Science. 309:481-484.

20. Chang, S., et al. 2004. Essential role of limiting telomeres in the pathogenesis of Werner syndrome. Nat. Genet. 36:877-882.

21. Kim, K.H., et al. 2004. Expression of connective tissue growth factor, a biomarker in senescence of human diploid fibroblasts, is up-regulated by a transforming growth factor-beta-mediated signaling pathway. Biochem. Biophys. Res. Commun. 318:819-825.

22. Hishikawa, K., et al. 1999. Connective tissue growth factor induces apoptosis in human breast cancer cell line MCF-7. J. Biol. Chem. 274:37461-37466.
23. Cordenonsi, M., et al. 2003. Links between tumor suppressors: p53 is required for TGF-beta gene responses by cooperating with Smads. Cell. 113:301-314.

24. Gore-Hyer, E., et al. 2002. TGF-beta and CTGF have overlapping and distinct fibrogenic effects on human renal cells. Am. J. Physiol. Renal Physiol. 283:F707-F716.

25. Seoane, J., Le, H.V., Shen, L., Anderson, S.A., and Massague, J. 2004. Integration of Smad and forkhead pathways in the control of neuroepithelial and glioblastoma cell proliferation. Cell. 117:211-223.

26. Kalivendi, S.V., et al. 2004. Alpha-synuclein upregulation and aggregation during $\mathrm{MPP}+$-induced apoptosis in neuroblastoma cells: intermediacy of transferrin receptor iron and hydrogen peroxide. J. Biol. Chem. 279:15240-15247.

27. Lesuisse, C., and Martin, L.J. 2002. Long-term culture of mouse cortical neurons as a model for neuronal development, aging, and death. J. Neurobiol. 51:9-23.

28. Machida, S., and Booth, F.W. 2004. Increased nuclear proteins in muscle satellite cells in aged animals as compared to young growing animals. Exp. Gerontol. 39:1521-1525.

29. Fraga, C.G., Shigenaga, M.K., Park, J.W., Degan, P., and Ames, B.N. 1990. Oxidative damage to DNA during aging: 8-hydroxy-2'-deoxyguanosine in rat organ DNA and urine. Proc. Natl. Acad. Sci. U. S. A. 87:4533-4537.

30. Michikawa, Y., Mazzucchelli, F., Bresolin, N., Scarlato, G., and Attardi, G. 1999. Aging-dependent large accumulation of point mutations in the human mtDNA control region for replication. Science. 286:774-779.

31. Tanhauser, S.M., and Laipis, P.J. 1995. Multiple deletions are detectable in mitochondrial DNA of aging mice. J. Biol. Chem. 270:24769-24775.

32. Khaidakov, M., Heflich, R.H., Manjanatha, M.G., Myers, M.B., and Aidoo, A. 2003. Accumulation of point mutations in mitochondrial DNA of aging mice. Mutat. Res. 526:1-7.

33. Edgell, C.J., McDonald, C.C., and Graham, J.B. 1983. Permanent cell line expressing human factor VIII-related antigen established by hybridization. 
Proc. Natl. Acad. Sci. U. S. A. 80:3734-3737.

34. US Renal Data System. 2005. 2005 Annual data report: atlas of end-stage renal disease in the United States. NIH, NIDDK. Bethesda, Maryland, USA.

35. Cessac, A.L., Perichon, M., Schaeverbeke, J., and Bakala, H. 1993. Age-related changes in albumin binding by renal brush-border membrane vesicles. Mech. Ageing Dev. 70:139-148.

36. Croft, P., and Hannaford, P.C. 1989. Risk factors for acute myocardial infarction in women: evidence from the Royal College of General Practitioners' oral contraception study. BMJ. 298:165-168.

37. Coughlin, S.S., Calle, E.E., Teras, L.R., Petrelli, J., and Thun, M.J. 2004. Diabetes mellitus as a predictor of cancer mortality in a large cohort of US adults. Am. J. Epidemiol. 159:1160-1167.

38. Albert, C.M., et al. 2003. Prospective study of sudden cardiac death among women in the United States. Circulation. 107:2096-2101.

39. Stegmayr, B., and Asplund, K. 1995. Diabetes as a risk factor for stroke. A population perspective. Diabetologia. 38:1061-1068.

40. Ott, A., et al. 1999. Diabetes mellitus and the risk of dementia: The Rotterdam Study. Neurology. 53:1937-1942.

41. Vestergaard, P., Rejnmark, L., and Mosekilde, L. 2005. Relative fracture risk in patients with diabetes mellitus, and the impact of insulin and oral antidiabetic medication on relative fracture risk. Diabetologia. 48:1292-1299.

42. Zendehdel, K., et al. 2003. Cancer incidence in patients with type 1 diabetes mellitus: a population-based cohort study in Sweden. J. Natl. Cancer Inst. 95:1797-1800.
43. Voutilainen-Kaunisto, R.M., Terasvirta, M.E., Uusitupa, M.I., and Niskanen, L.K. 2000. Age-related macular degeneration in newly diagnosed type 2 diabetic patients and control subjects: a 10-year follow-up on evolution, risk factors, and prognostic significance. Diabetes Care. 23:1672-1678.

44. Matsumoto, T., Ohashi, Y., Yamada, N., and Kikuchi, M. 1994. Hyperglycemia as a major determinant of distal polyneuropathy independent of age and diabetes duration in patients with recently diagnosed diabetes. Diabetes Res. Clin. Pract. 26:109-113.

45. Gurley, S.B., et al. 2006. Impact of genetic background on nephropathy in diabetic mice. Am.J. Physiol. Renal Physiol. 290:F214-F222.

46. Harman, D. 1956. Aging: a theory based on free radical and radiation chemistry. J. Gerontol. 11:298-300.

47. Brownlee, M. 2005. The pathobiology of diabetic complications. Diabetes. 54:1615-1625.

48. Hsu, G.W., Ober, M., Carell, T., and Beese, L.S. 2004. Error-prone replication of oxidatively damaged DNA by a high-fidelity DNA polymerase. Nature. 431:217-221.

49. Van Remmen, H., et al. 2003. Life-long reduction in MnSOD activity results in increased DNA damage and higher incidence of cancer but does not accelerate aging. Physiol. Genomics. 16:29-37.

50. Trifunovic, A., et al. 2004. Premature ageing in mice expressing defective mitochondrial DNA polymerase. Nature. 429:417-423.

51. Yasunari, K., et al. 2003. Converting enzyme inhibitor temocaprilat prevents high glucose-mediated suppression of human aortic endothelial cell proliferation. J. Cardiovasc. Pharmacol. 42(Suppl. 1):S55-S60.

52. Brown, G.C., Bolanos, J.P., Heales, S.J.R., and Clark,
J.B. 1995. Nitric oxide produced by activated astrocytes rapidly and reversibly inhibits cellular respiration. Neurosci. Lett. 193:201-204.

53. Loke, K.E., et al. 1999. Role of nitric oxide in the control of cardiac oxygen consumption in $\mathrm{B}(2)$-kinin receptor knockout mice. Hypertension. 34:563-567.

54. Adler, S., et al. 2001. Endothelial nitric oxide synthase plays an essential role in regulation of renal oxygen consumption by NO. Am. J. Physiol. Renal Physiol. 280:F838-F843.

55. Almeida, A., Moncada, S., and Bolanos, J.P. 2004. Nitric oxide switches on glycolysis through the AMP protein kinase and 6-phosphofructo-2-kinase pathway. Nat. Cell Biol. 6:45-51.

56. Schanstra, J.P., et al. 2003. Decreased renal NO excretion and reduced glomerular tuft area in mice lacking the bradykinin B2 receptor. Am. J. Physiol. Heart Circ. Physiol. 284:H1904-H1908.

57. Emanueli, C., et al. 1999. Dilated and failing cardiomyopathy in bradykinin $\mathrm{B}(2)$ receptor knockout mice. Circulation. 100:2359-2365.

58. Borkowski, J.A., et al. 1995. Targeted disruption of a $\mathrm{B} 2$ bradykinin receptor gene in mice eliminates bradykinin action in smooth muscle and neurons. J. Biol. Chem. 270:13706-13710.

59. Wang, J., et al. 1999. A mutation in the insulin 2 gene induces diabetes with severe pancreatic betacell dysfunction in the Mody mouse. J. Clin. Invest. 103:27-37.

60. Lapenna, D., Ciofani, G., Pierdomenico, S.D., Giamberardino, M.A., and Cuccurullo, F. 2001. Reaction conditions affecting the relationship between thiobarbituric acid reactivity and lipid peroxides in human plasma. Free Radic. Biol. Med. 31:331-335. 